\title{
Assessment of Roundup Ready 2 (RR2) corn subjected to application of isolated and associated herbicides
}

\section{Alfredo Junior Paiola Albrecht**1, Leandro Paiola Albrecht ${ }^{1}$, Fábio Henrique Krenchinski ${ }^{1}$, Katle Samaya Wobeto ${ }^{1}$, Mateus Dalpubel Mattiuzzi ${ }^{1}$, Caroline Thaís Eckert ${ }^{2}$, Caio Antonio Carbonari ${ }^{3}$, Ricardo Victoria Filho ${ }^{4}$} ${ }^{1}$ Federal University of Parana, Palotina Campus, Pioneer Street, 2153 - Garden Dallas, Palotina - PR, CEP:
85950-000, Brazil

${ }^{2}$ State University of the Western of Paraná, University Street, 2069 - University Garden, Cascavel - PR, CEP: 85819-110, Brazil

${ }^{3}$ State University of São Paulo “Júlio de Mesquita Filho”. Dr. José Barbosa de Barros Street, 1780, CEP: 18610307, Brazil

${ }^{4}$ University of São Paulo/ "Luiz de Queiroz" College of Agriculture, Padua Dias Avenue, 11 - Agronomy, Piracicaba - SP, CEP: 13418-900, Brazil

*Corresponding author: ajpalbrecht@yahoo.com.br

\begin{abstract}
One of the first transgenic traits that has been inserted into commercial crops and being widely used is tolerance to glyphosate herbicide. This technology has provided significant benefits and conveniences to farmers due to the efficacy of glyphosate. However, for a better weed control and prevention of weed resistant biotypes the use of two or more mechanisms is recommended practice. The combination of herbicides is the subject of several studies especially evaluating weed control, aside which the selectivity of the culture is equally important. In this context, this study aimed to evaluate the responses of transgenic corn (RR2) subjected to the application of different herbicides. For this purpose, an experiment was conducted with eight treatments using glyphosate, atrazine and nicosulfuron, sole or in combination along with a control treatment (without herbicide). The experiment was performed twice (first in Piracicaba - SP then Palotina - PR) in greenhouse with different corn hybrids. Several variables related to the performance were analysed such as height measurements, stem diameter, chlorophyll index, fresh and dry shoot, root dry mass. The results showed that nicosulfuron should be positioned cautiously in new RR2 corn hybrids, as it may damage the development of the plant.
\end{abstract}

Keywords: Zea mays, Transgenic Crops, Selectivity, Roundup Ready 2 Technology.

Introduction

In Brazil, corn is surpassed by soybean crop in production area and grain productivity. According to Conab (2015), in 2014/2015 harvest season, the total area of corn (first and second season) was about 15.7 million hectares with an average productivity of 5,396 $\mathrm{kg} \mathrm{ha}^{-1}$. In 2015/2016 season, the estimated total area of corn (first and second season) is about 15.3 million hectares and the average yield will be $5370 \mathrm{~kg} \mathrm{ha}^{-1}(63 \%$ of this area and $66 \%$ of production correspond to the second corn crop).

The second generation of RR corn has an enzyme (EPSPs) different from the first. The NK603 (RR2) corn was developed using viral promoters and regulatory elements that increase the expression of tolerance to glyphosate (N(fosfonometil)glicina) in plant, especially in previously vulnerable tissues (CaJacob et al., 2007). The homology of this enzyme with wild EPSPs is about $99.7 \%$ according to the authors (homology with the EPSPs of the A. tumefaciens bacteria). This change has remedied the previous problem of phytointoxication, and also allowed Monsanto to renew its patents and introduce it into the market as a material with higher acceptability by producers. In Brazil the first commercial hybrid corn, tolerant to glyphosate includes second generation of RR technology. The hybrid name is
NK603 - Roundup Ready 2 (CIB, 2016).

In 2014/2015 harvest season, the adoption of biotechnology for corn totaled $83 \%$, considering the planted corn area of the first and second harvest. In this scenario, 53\% of areas were grown with hybrids that showed tolerance to glyphosate. Also, the tolerance to this herbicide associated with $92 \%$ of cases to various events that confer resistance to insects (Celeres, 2015).

The percentage of the areas cultivated with glyphosate tolerant corn and other herbicides will grow significantly due to the increased supply of hybrids, the larger amount of seeds available on the market and the new technologies offered, which show tolerance to different herbicides (Borém et al., 2015).

In the world, there are currently 32 registered species of weeds resistant to glyphosate. In the United States, there are 16 species of weeds resistant to glyphosate; Australia has 10; and Brazil has eight species resistant to glyphosate (Heap, 2016). Results from Shaner (2000) warned that although the crops tolerant to glyphosate are important tools in weed control, intensive use of glyphosate in RR crops would cause problems, especially in the selection of resistant biotypes. In this sense, focusing on a better control of weeds and 
preventing the selection of resistant weed biotypes, the use of two or more mechanisms of action are advisable measures (Riar et al., 2013; Barroso et al., 2015). A recent survey by Gazziero (2015) found that for $97 \%$ of rural and technical producers, tank-mixing is a common practice in $95 \%$ of the times ranging from two to five products and that $72 \%$ of the interviewed producers said they did not know anything or have used insufficient amount of information about tank mixtures.

Herbicide associations are often studied to control weeds that are hard to manage (Azevedo, 2015; Gemelli, et al., 2013; Melo et al., 2012). But selectivity of the crops cannot be left aside. The product associations are very important because even if the weed control is effective the culture of economic interest may be adversely affected by the applied products and farmers will still be negatively affected in some ways.

With the expansion of RR2 corn, doubts have arisen concerning the association of glyphosate with other herbicides, which are widely used in corn even before enter of transgenic plants. There is the risk of causing phytointoxication in the culture or undermining the effectiveness of weed control. These questions denote the needs for further studies in these areas. Brazilian agricultural regions have displayed some cases of antagonism to herbicide associations applied in crops such as soybeans and corn, resulting in visible phytointoxications (Albrecht et al., 2014).

Due to this fact, it is necessary to search for information enabling a diagnosis of negative consequences related to the use of glyphosate and other herbicides applied alone or associated in RR2 corn, and to encourage more sustainable and secure positioning of this technology in new hybrids, providing benefits to farmers for proper weed management in the agro-ecosystem without harming the culture of economic interest. Thus, this study aimed to evaluate the potential impacts of the application of glyphosate, atrazine (2-chloro4-ethylamino-6-isopropylamino-1,3,5-triazine) and nicosulfuron (2-(4,6-dimethoxypyrimidin-2-ylcarbamoyl sulfamoyl)-N,N-dimethylnicotinamide), isolated (sole) and associated (in combination) at post-emergence stages of RR2 corn.

\section{Results and Discussion}

\section{Experiment performed in Piracicaba - SP (2014)}

For the first experiment conducted in Piracicaba - SP, there was no difference at 14 DAA for plant height (Table 3). The plant height was shorter in treatments 6 and 7 at 28 DAA, which highlights the fact that treatments 6 and 7 present less height than treatments 2 and 3 .

In Table 5, the results of the SPAD index displayed difference for the measurement made at 14 DAA, in which treatment 6 showed an index lower than treatment 1 . Table 7 shows that no differences are observed in stem diameter. The other four variables are related to the mass demonstrated differences.

Starting with the assessment of fresh mass of the shoot, there is a similar behavior to what is seen in the height evaluation at 28 DAA (Table 6). For dry matter of the shoot, it was seen that treatments 6 and 7 expressed smaller masses than treatments $1,2,3$ and 4 . For the dry mass of the plant root system, it is emphasized that treatments 7 and 8 had lower masses than treatments 3 and 4 . In the total dry weight assessment, treatments 6 and 7 had less mass than treatments $1,2,3$ and 4 , since treatment 8 had lower mass than treatments 2 and 3 .

Considering treatments 6 and 8 , it is understood that the product that causes major problem in association with other herbicides is nicosulfuron We concluded that when this herbicide is used isolated (treatment 4) and particularly when associated with glyphosate (treatment 6) tends to damage the RR2 corn. This hybrid corn is a simple hybrid and being used for the first time in this experiment.

Upon interpretation of these results, a solid and strong fact was observed that nicosulfuron expressed possible damage to plants, even used alone or in combination. This is related to differential sensitivity of the corn hybrid to this herbicide, similar to the effects discussed in the work with conventional corn (Pereira Filho et al., 2000; Vargas et al., 2006; Cavalieri et al., 2008; Cavalieri et al., 2010; Dan et al., 2011).

Mainly for the variables related to height and biomass accumulation, the problem with nicosulfuron becomes evident. The point is that nicosulfuron has some negative effects and associations with many even it may or may not impose damage to the plant. However, it is emphasized that glyphosate and atrazine, isolated or associated, did not affect the development of culture.

\section{Experiment performed in Palotina - PR (2015)}

In the second experiment, which was conducted in Palotina PR (2015), the same treatments were applied. The altered factor was the hybrid corn, which had very different characteristics compared to the previous.

For plant height assessments (Table 7), the only difference was in the assessment at 21 DAA, in which treatments 2 and 4 had less height than treatment 8 . Thus, the crop's response to the applied treatments was different from what was seen at the first experiment.

Evaluations of plant stem diameter (Table 8) showed that the only difference observed at 28 DAA, in which treatment 6 had a smaller diameter than treatment 2 , verifying a partial match with the results of previous experiments.

For chlorophyll A indexes (Table 9), chlorophyll B (Table 10) and total chlorophyll (Table 11), assessed at 7, 14, 21 and 28 DAA, there was no difference between the treatments. Similarly, for mass evaluations (Table 12), no differences were displayed between the treatments.

A due care is needed to compare the experiments, because a conjoint analysis was not employed, but going for a relationship between them, some differentiated behaviors of the treatments were visible in regard to the first and second experiments were performed. This may have occurred due to the hybrid corn that used in each experiment which was different between the two tests. This fact is indicative of the difference in the plant response in accordance with the genotype used according to Cavalieri et al. (2010). It may depend on the hybrid origin, which may be more or less tolerant to nicosulfuron. It can be justified by the greater or lesser capacity of the hybrid to absorb, metabolize and translocate the molecule.

The nicosulfuron caused damage to the first hybrid at the first experiment. However, in the second hybrid (of second experiment) this effect was not observed likewise, probably due to differential sensitivity or tolerance between genotypes to this herbicide. 
Table 1. Treatments of herbicides applied isolated or associated (sole or in combination) on RR2 corn. Greenhouse, Pricacicaba - SP (2014) and Palotina - PR (2015).

\begin{tabular}{|c|c|c|}
\hline & Treatments & Doses* \\
\hline $\mathrm{T} 1$ & Control & 0 \\
\hline $\mathrm{T} 2$ & Glyphosate & $1080\left(\right.$ g a.e. ha $\left.^{-1}\right)$ \\
\hline $\mathrm{T} 3$ & Atrazine & $2000\left(\mathrm{~g}\right.$ a.i. $\left.\mathrm{ha}^{-1}\right)$ \\
\hline $\mathrm{T} 4$ & Nicosulfuron & $50\left(\mathrm{~g}\right.$ a.i. ha $\left.{ }^{-1}\right)$ \\
\hline T5 & Glyphosate + atrazine & $1080\left(\right.$ g a.e. ha $\left.^{-1}\right)+2000\left(\right.$ g a.i. ha $\left.{ }^{-1}\right)$ \\
\hline T6 & Glyphosate + nicosulfuron & $1080\left(\mathrm{~g}\right.$ a.e. ha $\left.{ }^{-1}\right)+50\left(\mathrm{~g}\right.$ a.i. $\left.\mathrm{ha}^{-1}\right)$ \\
\hline $\mathrm{T} 7$ & Atrazine + nicosulfuron & $2000\left(\mathrm{~g}\right.$ a.i. $\left.\mathrm{ha}^{-1}\right)+50\left(\mathrm{~g}\right.$ a.i. $\left.\mathrm{ha}^{-1}\right)$ \\
\hline $\mathrm{T} 8$ & $\begin{array}{l}\text { Glyphosate + atrazine + } \\
\text { Nicosulfuron }\end{array}$ & 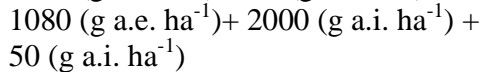 \\
\hline
\end{tabular}

$*_{\mathrm{g}}$ a.e. $\mathrm{ha}^{-1}=$ grams of active ingredient per hectare.

Table 2. Result of chemical and physical analysis of the soil used for the experiment in pots.

\begin{tabular}{|c|c|c|c|c|c|c|c|c|c|}
\hline \multirow{4}{*}{ 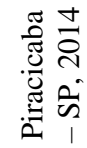 } & $\mathrm{P}($ Resin) & $\mathrm{K}$ & $\mathrm{C}$ & $\mathrm{Ca}$ & $\mathrm{Mg}$ & $\mathrm{H}+\mathrm{Al}$ & $\mathrm{pH}\left(\mathrm{CaCl}_{2}\right)$ & $\mathrm{Al}$ & SB \\
\hline & 7.0 & 0.26 & 10.27 & 3.90 & 1.60 & 2.50 & 5.3 & 0.00 & 5.76 \\
\hline & $\mathrm{Zn}$ & $\mathrm{Fe}$ & $\mathrm{Mn}$ & $\mathrm{Cu}$ & $\mathrm{V}$ & CEC & Sand & Silt & Clay \\
\hline & 2.6 & 33.0 & 7.3 & 1.6 & 70.0 & 82.8 & 54.00 & 6.00 & 40.00 \\
\hline \multirow{4}{*}{ 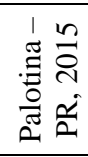 } & P (Mehlich) & $\mathrm{K}$ & $\mathrm{C}$ & $\mathrm{Ca}$ & $\mathrm{Mg}$ & $\mathrm{H}+\mathrm{Al}$ & $\mathrm{pH}\left(\mathrm{CaCl}_{2}\right)$ & $\mathrm{Al}$ & SB \\
\hline & 8.93 & 0.51 & 13.65 & 5.39 & 0.87 & 4.28 & 5.5 & 0.00 & 6.77 \\
\hline & $\mathrm{Zn}$ & $\mathrm{Fe}$ & $\mathrm{Mn}$ & $\mathrm{Cu}$ & $\mathrm{V}$ & CEC & Sand & Silt & Clay \\
\hline & 1.44 & 19.59 & 39.12 & 10.01 & 61.27 & 11.05 & 17.75 & 16.50 & 65.75 \\
\hline
\end{tabular}

Units: $\mathrm{P}, \mathrm{Cu}, \mathrm{Zn}, \mathrm{Fe}, \mathrm{Mn}\left(\mathrm{mg} \mathrm{dm}{ }^{-3}\right) ; \mathrm{K}, \mathrm{Ca}, \mathrm{Mg}, \mathrm{H}+\mathrm{Al}, \mathrm{Al}, \mathrm{SB}, \mathrm{CEC}\left(\mathrm{cmol}_{\mathrm{c}} \mathrm{dm}^{-3}\right) ; \mathrm{C}\left(\mathrm{g} \mathrm{dm}^{-3}\right) ; \mathrm{V}$, sand, silt, clay $(\%)$

Table 3. Measurements of height (cm) of corn plants RR2 (30A91HR), 7, 14, 21 and 28 days after application (DAA) of eight treatments comprised of isolated and associated herbicides. Piracicaba - SP, 2014.

\begin{tabular}{lcccc}
\hline Treatments & Height 7 DAA* & Height 14 DAA & Height 21 DAA* & Height 28 DAA \\
\hline T1 & 24.00 & $32.75 \mathrm{ab}$ & 42.75 & $57.00 \mathrm{abc}$ \\
T2 & 25.00 & $33.50 \mathrm{ab}$ & 47.00 & $61.50 \mathrm{a}$ \\
T3 & 27.50 & $35.75 \mathrm{a}$ & 47.00 & $61.75 \mathrm{a}$ \\
T4 & 26.75 & $34.00 \mathrm{ab}$ & 46.25 & $60.75 \mathrm{ab}$ \\
T5 & $32.00 \mathrm{ab}$ & 41.75 & $54.25 \mathrm{abc}$ \\
T6 & $30.00 \mathrm{~b}$ & 43.25 & $53.00 \mathrm{bc}$ \\
T7 & $30.25 \mathrm{~b}$ & 39.00 & $52.50 \mathrm{c}$ \\
T8 & 23.75 & $31.50 \mathrm{ab}$ & 41.00 & $56.50 \mathrm{abc}$ \\
\hline Average & 22.75 & 32.47 & 43.50 & 57.16 \\
\hline CV\% & 24.75 & 7.06 & 9.90 & 5.97 \\
\hline LSD & 25.09 & 5.37 & 10.09 & 7.99 \\
\hline
\end{tabular}

Averages followed by same letter in the column do not differ significantly $(P<0.05)$ by Tukey test.

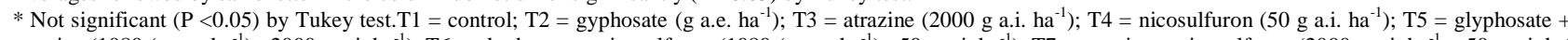
atrazine $\left(1080\left(\mathrm{~g}\right.\right.$ a.e. ha $\left.{ }^{-1}\right)+2000 \mathrm{~g}$ a.i. ha $\left.{ }^{-1}\right) ; \mathrm{T} 6=$ glyphosate + nicosulfuron $\left(1080\left(\mathrm{~g}\right.\right.$ a.e. $\left.\mathrm{ha}^{-1}\right)+50 \mathrm{~g}^{-1}$.i. ha $\left.{ }^{-1}\right) ; \mathrm{T} 7=$ atrazine + nicosulfuron $\left(2000 \mathrm{~g}\right.$ a.i. ha ${ }^{-1}+50 \mathrm{~g}$ a.i. ha

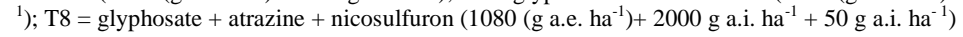

Table 4. SPAD index (Minolta unit) of leaves of maize RR2 (30A91HR), 7, 14, 21 and 28 days after application (DAA) of eight treatments comprised isolates and associated herbicides. Piracicaba - SP, 2014.

\begin{tabular}{lcccc}
\hline Treatments & SPAD 7 DAA & SPAD 14 DAA & SPAD 21 DAA* & SPAD 28 DAA* \\
\hline T1 & 35.30 & $38.77 \mathrm{a}$ & 36.45 & 38.41 \\
T2 & 36.60 & $36.45 \mathrm{ab}$ & 37.95 & 41.37 \\
T3 & 37.07 & $37.70 \mathrm{ab}$ & 36.52 & 39.15 \\
T4 & 35.02 & $37.90 \mathrm{ab}$ & 37.47 & 36.17 \\
T5 & 34.92 & $35.55 \mathrm{ab}$ & 36.72 & 40.77 \\
T6 & 33.52 & $34.80 \mathrm{~b}$ & 33.65 & 37.85 \\
T7 & 32.85 & $37.65 \mathrm{ab}$ & 34.95 & 36.52 \\
T8 & 36.62 & 36.52 & 35.90 & 38.20 \\
\hline Average & 35.26 & 5.76 & 36.24 & 8.91 \\
\hline CV\% & 7.62 & 4.93 & 5.32 & 7.97 \\
\hline LSD & 6.31 & & 4.51 & \\
\hline
\end{tabular}

Averages followed by same letter in the column do not differ significantly $(P<0.05)$ by Tukey test.

* Not significant $(\mathrm{P}<0.05)$ by Tukey test. T1 = control; $\mathrm{T} 2=$ gyphosate $\left(1080\left(\mathrm{~g}\right.\right.$ a.e. $\left.\left.\mathrm{ha}^{-1}\right)\right)$; T3 = atrazine $\left(2000 \mathrm{~g}\right.$ a.i. ha $\left.{ }^{-1}\right)$; $\mathrm{T} 4=$ nicosulfuron $\left(50 \mathrm{~g}\right.$ a.i. ha $\left.{ }^{-1}\right)$; $\mathrm{T} 5=$ glyphosate + atrazine $\left(1080\left(\mathrm{~g}\right.\right.$ a.e. $\left.\mathrm{ha}^{-1}\right)+2000 \mathrm{~g}$ a.i. $\left.\mathrm{ha}^{-1}\right) ; \mathrm{T} 6=$ glyphosate + nicosulfuron $\left(1080\left(\mathrm{~g}\right.\right.$ a.e. ha $\left.{ }^{-1}\right)+50 \mathrm{~g}$ a.i. ha $\left.{ }^{-1}\right) ; \mathrm{T} 7=\mathrm{atrazine}^{-1}$ nicosulfuron $\left(2000 \mathrm{~g}\right.$ a.i. ha ${ }^{-1}$ $+50 \mathrm{~g}$ a.i. ha $\left.{ }^{-1}\right) ; \mathrm{T} 8=$ glyphosate + atrazine + nicosulfuron $\left(1080\left(\mathrm{~g}\right.\right.$ a.e. $\left.\mathrm{ha}^{-1}\right)+2000 \mathrm{~g}$ a.i. $\mathrm{ha}^{-1}+50 \mathrm{~g}$ a.i. ha $\left.{ }^{-1}\right)$ 
Table 5. Measurements of stem diameter (mm), fresh weight of aerial parts $(\mathrm{g})$, dry mass of leave (g) root dry mass $(\mathrm{g})$ and total dry mass (g) of RR2 maize (30A91HR), at 28 day after the application of eight treatments comprised isolates and associated herbicides. Piracicaba - SP, 2014.

\begin{tabular}{|c|c|c|c|c|c|}
\hline Treatments & Stem Diameter* & $\begin{array}{l}\text { Fresh Weight of } \\
\text { Aerial Parts }\end{array}$ & Dry Mass of Leave & Root Dry Mass & Total Dry Mass \\
\hline $\mathrm{T} 1$ & 17.25 & $115.26 \mathrm{abc}$ & $23.18 \mathrm{a}$ & 9.38 abc & $32.57 \mathrm{ab}$ \\
\hline $\mathrm{T} 2$ & 18.25 & $122.12 \mathrm{a}$ & $24.70 \mathrm{a}$ & $9.86 \mathrm{abc}$ & $34.56 \mathrm{a}$ \\
\hline T3 & 16.25 & $125.49 \mathrm{a}$ & $23.84 \mathrm{a}$ & $10.60 \mathrm{a}$ & $34.45 \mathrm{a}$ \\
\hline $\mathrm{T} 4$ & 15.50 & $117.11 \mathrm{ab}$ & $22.64 \mathrm{a}$ & $10.36 \mathrm{ab}$ & $33.01 \mathrm{ab}$ \\
\hline T5 & 15.75 & $104.65 \mathrm{abc}$ & $20.44 \mathrm{ab}$ & $8.53 \mathrm{bcd}$ & $28.98 \mathrm{abc}$ \\
\hline T6 & 14.75 & $84.57 \mathrm{bc}$ & $15.52 b$ & $8.62 \mathrm{abcd}$ & $24.14 \mathrm{c}$ \\
\hline $\mathrm{T} 7$ & 14.00 & $79.83 \mathrm{c}$ & $14.14 \mathrm{~b}$ & $8.14 \mathrm{~cd}$ & $22.28 \mathrm{c}$ \\
\hline $\mathrm{T} 8$ & 16.75 & $99.28 \mathrm{abc}$ & $18.74 \mathrm{ab}$ & $7.10 \mathrm{~d}$ & $25.84 \mathrm{bc}$ \\
\hline Average & 16.06 & 106.04 & 20.40 & 9.07 & 29.48 \\
\hline $\mathrm{CV} \%$ & 16.44 & 14.82 & 13.34 & 9.63 & 11.14 \\
\hline LSD & 6.23 & 36.82 & 6.38 & 2.05 & 7.69 \\
\hline
\end{tabular}

Table 6. Measurement height (cm) of corn plants RR2/LL (30F53HR), 7, 14, 21 and 28 days after application (DAA) of eight treatments consisting of isolated and associates herbicides. Palotina - PR, 2015.

\begin{tabular}{|c|c|c|c|c|}
\hline Treatments & Height 7 DAA* & Height $14 \mathrm{DAA}^{*}$ & Height 21 DAA & Height 28 DAA* \\
\hline $\mathrm{T} 1$ & 33.75 & 45.50 & $57.50 \mathrm{ab}$ & 73.00 \\
\hline $\mathrm{T} 2$ & 34.50 & 40.75 & $56.00 \mathrm{~b}$ & 72.50 \\
\hline $\mathrm{T} 3$ & 38.00 & 46.25 & $59.50 \mathrm{ab}$ & 76.50 \\
\hline $\mathrm{T} 4$ & 35.25 & 42.00 & $54.50 \mathrm{~b}$ & 79.75 \\
\hline T5 & 35.50 & 48.75 & $61.00 \mathrm{ab}$ & 75.25 \\
\hline T6 & 35.75 & 46.50 & $60.00 \mathrm{ab}$ & 77.25 \\
\hline $\mathrm{T} 7$ & 34.00 & 47.50 & $60.50 \mathrm{ab}$ & 70.75 \\
\hline $\mathrm{T} 8$ & 35.00 & 50.50 & $64.50 \mathrm{a}$ & 74.00 \\
\hline Average & 35.22 & 45.97 & 59.19 & 74.88 \\
\hline $\mathrm{CV} \%$ & 6.77 & 9.25 & 5.68 & 6.39 \\
\hline LSD & 5.58 & 9.96 & 7.87 & 11.20 \\
\hline
\end{tabular}

Table 7. Measurement of stem diameter (mm) of corn plants RR2/LL (30F53HR), 7, 14, 21 and 28 days after application (DAA) of eight treatments consisting of isolated and associated herbicides. Palotina - PR, 2015.

\begin{tabular}{|c|c|c|c|c|}
\hline Treatments & $\begin{array}{l}\text { Diameter } \\
7 \text { DAA* }\end{array}$ & $\begin{array}{l}\text { Diameter } \\
14 \text { DAA* }^{*}\end{array}$ & $\begin{array}{l}\text { Diameter } \\
21 \mathrm{DAA}^{*}\end{array}$ & $\begin{array}{c}\text { Diameter } \\
28 \text { DAA }\end{array}$ \\
\hline $\mathrm{T} 1$ & 11.03 & 14.93 & 15.68 & $16.78 \mathrm{ab}$ \\
\hline $\mathrm{T} 2$ & 11.68 & 15.02 & 16.47 & $18.14 \mathrm{a}$ \\
\hline $\mathrm{T} 3$ & 11.95 & 15.53 & 16.09 & $16.87 \mathrm{ab}$ \\
\hline $\mathrm{T} 4$ & 10.91 & 15.20 & 15.60 & $16.95 \mathrm{ab}$ \\
\hline $\mathrm{T} 5$ & 11.48 & 14.90 & 15.24 & $16.67 \mathrm{ab}$ \\
\hline T6 & 11.12 & 14.32 & 14.80 & $15.67 \mathrm{~b}$ \\
\hline $\mathrm{T} 7$ & 10.78 & 14.61 & 15.53 & $16.41 \mathrm{ab}$ \\
\hline $\mathrm{T} 8$ & 11.73 & 14.80 & 15.19 & $15.87 \mathrm{ab}$ \\
\hline Average & 11.34 & 14.91 & 15.57 & 16.67 \\
\hline $\mathrm{CV} \%$ & 6.62 & 5.11 & 5.61 & 6.10 \\
\hline LSD & 1.76 & 1.79 & 2.05 & 2.38 \\
\hline
\end{tabular}

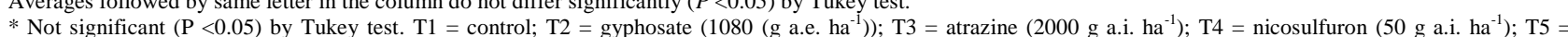

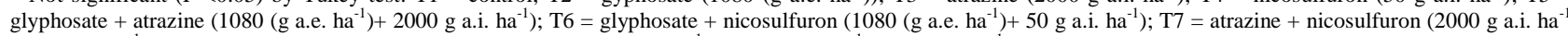
$+50 \mathrm{~g}$ a.i. ha $\left.{ }^{-1}\right) ; \mathrm{T} 8=$ glyphosate + atrazine + nicosulfuron $\left(1080\left(\mathrm{~g}\right.\right.$ a.e. $\left.\mathrm{ha}^{-1}\right)+2000 \mathrm{~g}$ a.i. $\mathrm{ha}^{-1}+50 \mathrm{~g}^{-1}$.i. ha $\left.{ }^{-1}\right)$ 
Table 8. Chlorophyll index Falker (ICF) determining Chlorophyll A (Falker unit), in leaves of corn RR2/LL (30F53HR), evaluated 7, 14, 21 and 28 days after the application of eight treatments comprised isolated and associated herbicides. Palotina - PR, 2015.

\begin{tabular}{lcccc}
\hline Treatments & $\begin{array}{c}\text { Chlorophyll A } \\
\text { 7 DAA* }\end{array}$ & $\begin{array}{c}\text { Chlorophyll A } \\
\text { 14 DAA* }\end{array}$ & $\begin{array}{c}\text { Chlorophyll A } \\
\text { 21 DAA* }\end{array}$ & $\begin{array}{c}\text { Chlorophyll A } \\
\text { 28 DAA* }\end{array}$ \\
\hline T1 & 34.60 & 28.40 & 36.87 & 29.95 \\
T2 & 34.65 & 29.45 & 34.97 & 27.67 \\
T3 & 35.22 & 27.58 & 35.27 & 30.20 \\
T4 & 34.65 & 28.82 & 36.32 & 30.82 \\
T5 & 35.15 & 31.30 & 37.77 & 26.72 \\
T6 & 36.27 & 29.40 & 34.97 & 27.92 \\
T7 & 36.37 & 29.62 & 36.77 & 29.32 \\
T8 & 37.80 & 27.67 & 37.42 & 28.57 \\
\hline Average & 35.60 & 29.03 & 36.30 & 28.90 \\
\hline CV\% & 7.77 & 8.37 & 5.76 & 9.47 \\
\hline LSD & 6.48 & 5.69 & 4.89 & 6.41 \\
\hline Averages followed by same letter in the column do not differ significantly $(P<0.05)$ by Tukey test & & \\
\hline
\end{tabular}

$*$ Not significant $(\mathrm{P}<0.05)$ by Tukey test. $\mathrm{T} 1=$ control; $\mathrm{T} 2=$ gyphosate $\left(1080\left(\mathrm{~g}\right.\right.$ a.e. $\left.\left.\mathrm{ha}^{-1}\right)\right)$; $\mathrm{T} 3=$ atrazine $\left(2000 \mathrm{~g}\right.$ a.i. ha $\left.{ }^{-1}\right)$; $\mathrm{T} 4=$ nicosulfuron $\left(50 \mathrm{~g}\right.$ a.i. ha $\left.{ }^{-1}\right)$; $\mathrm{T} 5=$

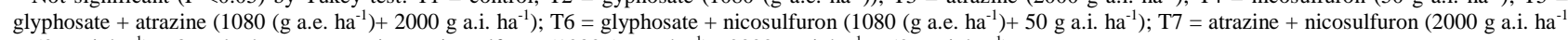
$+50 \mathrm{~g}$ a.i. $\left.\mathrm{ha}^{-1}\right) ; \mathrm{T} 8=$ glyphosate + atrazine + nicosulfuron $\left(1080\left(\mathrm{~g}\right.\right.$ a.e. $\left.\mathrm{ha}^{-1}\right)+2000 \mathrm{~g}$ a.i. $\left.\mathrm{ha}^{-1}+50 \mathrm{~g}^{-1} . \mathrm{ha}^{-1}\right)$

Table 9. Chlorophyll index Falker (ICF) determining Chlorophyll B (Falker unit). in leaves of corn RR2/LL (30F53HR). evaluated at 7, 14, 21 and 28 days after the application of eight treatments comprised of isolated and associated herbicides. Palotina - PR. 2015.

\begin{tabular}{lcccc}
\hline Treatments & $\begin{array}{c}\text { Chlorophyll B } \\
\text { 7 DAA* }\end{array}$ & $\begin{array}{c}\text { Chlorophyll B } \\
\text { 14 DAA* }\end{array}$ & $\begin{array}{c}\text { Chlorophyll B } \\
\text { 21 DAA* }\end{array}$ & $\begin{array}{c}\text { Chlorophyll B } \\
\text { 28 DAA* }\end{array}$ \\
\hline T1 & 7.82 & 5.00 & 9.25 & 5.80 \\
T2 & 8.77 & 5.30 & 8.80 & 4.72 \\
T3 & 8.77 & 5.35 & 8.20 & 5.57 \\
T4 & 8.07 & 5.17 & 8.72 & 6.17 \\
T5 & 8.52 & 6.62 & 9.60 & 4.80 \\
T6 & 9.10 & 5.65 & 8.32 & 5.70 \\
T7 & 8.70 & 5.95 & 8.67 & 5.55 \\
T8 & 9.37 & 5.07 & 9.37 & 5.27 \\
\hline Average & 8.64 & 5.52 & 8.87 & 5.45 \\
\hline CV\% & 10.13 & 12.63 & 10.16 & 12.89 \\
\hline LSD & 2.05 & 1.63 & 2.11 & 1.64 \\
\hline
\end{tabular}

Averages followed by same letter in the column do not differ significantly $(P<0.05)$ by Tukey test.

* Not significant $(\mathrm{P}<0.05)$ by Tukey test. $\mathrm{T} 1=$ control; $\mathrm{T} 2=$ gyphosate $\left(1080\left(\mathrm{~g}\right.\right.$ a.e. ha $\left.\left.{ }^{-1}\right)\right) ; \mathrm{T} 3=$ atrazine $\left(2000 \mathrm{~g}^{-1 . i}\right.$ ha $\left.{ }^{-1}\right) ; \mathrm{T} 4=$ nicosulfuron $\left(50 \mathrm{~g}\right.$ a.i. ha $\left.{ }^{-1}\right)$; $\mathrm{T} 5=$ glyphosate + atrazine $\left(1080\left(\mathrm{~g}_{\text {a.e. }} \mathrm{ha}^{-1}\right)+2000 \mathrm{~g}\right.$ a.i. ha $\left.\mathrm{a}^{-1}\right) ; \mathrm{T} 6=$ glyphosate + nicosulfuron $\left(1080\left(\mathrm{~g}\right.\right.$ a.e. ha $\left.\mathrm{g}^{-1}\right)+50 \mathrm{~g}$ a.i. ha $\left.{ }^{-1}\right) ; \mathrm{T} 7=$ atrazine + nicosulfuron $\left(2000 \mathrm{~g}\right.$ a.i. ha ${ }^{-1}$ $+50 \mathrm{~g}$ a.i. ha $\left.{ }^{-1}\right) ; \mathrm{T} 8=$ glyphosate + atrazine + nicosulfuron $\left(1080\left(\mathrm{~g}\right.\right.$ a.e. $\left.\mathrm{ha}^{-1}\right)+2000 \mathrm{~g}$ a.i. $\mathrm{ha}^{-1}+50 \mathrm{~g}$ a.i. ha $\left.{ }^{-1}\right)$

Table 10. Chlorophyll index Falker (ICF) calculated Chlorophyll Total (Falker unit). in leaves of corn RR2/LL (30F53HR). measured at 7. 14. 21 and 28 days after the application of eight treatments comprised isolates herbicides and associated. Palotina PR. 2015.

\begin{tabular}{lcccc}
\hline Treatments & $\begin{array}{c}\text { Chlorophyll Total } \\
\text { 7 DAA* }\end{array}$ & $\begin{array}{c}\text { Chlorophyll Total } \\
\text { 14 DAA* }\end{array}$ & $\begin{array}{c}\text { Chlorophyll Total } \\
\text { 21 DAA* }\end{array}$ & $\begin{array}{c}\text { Chlorophyll Total } \\
\text { 28 DAA* }\end{array}$ \\
\hline T1 & 42.43 & 33.43 & 46.13 & 35.75 \\
T2 & 43.43 & 34.75 & 43.78 & 32.40 \\
T3 & 44.00 & 33.00 & 43.48 & 35.78 \\
T4 & 42.73 & 34.00 & 45.05 & 37.00 \\
T5 & 43.68 & 37.93 & 47.38 & 31.53 \\
T6 & 45.38 & 35.05 & 43.30 & 33.63 \\
T7 & 45.08 & 35.65 & 45.45 & 34.88 \\
T8 & 47.18 & 32.75 & 46.80 & 33.85 \\
\hline Average & 44.23 & 34.57 & 45.17 & 34.35 \\
\hline CV\% & 7.71 & 8.53 & 6.30 & 9.67 \\
\hline LSD & 7.99 & 6.91 & 6.66 & 7.78 \\
\hline Av & & & & \\
\hline
\end{tabular}

Averages followed by same letter in the column do not differ significantly $(P<0.05)$ by Tukey test

* Not significant $(\mathrm{P}<0.05)$ by Tukey test. $\mathrm{T} 1=$ control; $\mathrm{T} 2=$ gyphosate $\left(1080\left(\mathrm{~g}\right.\right.$ a.e. ha $\left.\left.{ }^{-1}\right)\right)$; $\mathrm{T} 3=$ atrazine $\left(2000 \mathrm{~g}\right.$ a.i. ha $\left.{ }^{-1}\right) ; \mathrm{T} 4=$ nicosulfuron $\left(50 \mathrm{~g}\right.$ a.i. ha $\left.{ }^{-1}\right)$; T5 $=$

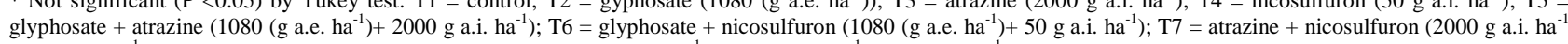
$+50 \mathrm{~g}$ a.i. ha $\left.{ }^{-1}\right) ; \mathrm{T} 8=$ glyphosate + atrazine + nicosulfuron $\left(1080\left(\mathrm{~g}\right.\right.$ a.e. $\left.\mathrm{ha}^{-1}\right)+2000 \mathrm{~g}$ a.i. $\mathrm{ha}^{-1}+50 \mathrm{~g}$ a.i. ha $\left.{ }^{-1}\right)$ 
Table 11. Fresh mass of the Aerial Part (g), Dry Mass of Aerial Part (g), Root Dry Mass (g) and Total Dry Mass, in corn RR2/LL (30F35HR) 28 days after the implementation of eight compounds treatments herbicides by isolates and associated. Palotina - PR. 2015 .

\begin{tabular}{|c|c|c|c|c|}
\hline Treatments & $\begin{array}{l}\text { Fresh Mass of the Aerial } \\
\text { Part* }\end{array}$ & $\begin{array}{l}\text { Dry Mass of Aerial } \\
\text { Part * }\end{array}$ & Root Dry Mass * & Total Dry Mass * \\
\hline $\mathrm{T} 1$ & 122.25 & 23.79 & 13.44 & 37.23 \\
\hline $\mathrm{T} 2$ & 126.75 & 23.30 & 12.14 & 35.44 \\
\hline $\mathrm{T} 3$ & 123.75 & 22.91 & 12.05 & 34.95 \\
\hline $\mathrm{T} 4$ & 123.00 & 23.07 & 13.32 & 36.39 \\
\hline T5 & 115.25 & 23.41 & 11.68 & 35.09 \\
\hline T6 & 119.75 & 23.45 & 12.60 & 36.05 \\
\hline $\mathrm{T} 7$ & 126.75 & 24.13 & 11.54 & 35.67 \\
\hline $\mathrm{T} 8$ & 117.25 & 23.54 & 13.11 & 36.65 \\
\hline Average & 121.84 & 23.45 & 12.48 & 35.93 \\
\hline $\mathrm{CV} \%$ & 6.88 & 7.70 & 14.99 & 7.65 \\
\hline LSD & 19.64 & 4.23 & 4.38 & 6.44 \\
\hline
\end{tabular}

Averages followed by same letter in the column do not differ significantly $(P<0.05)$ by Tukey test.

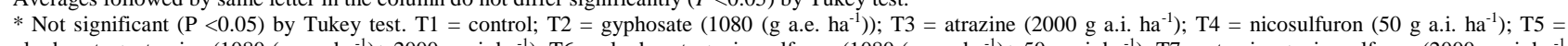

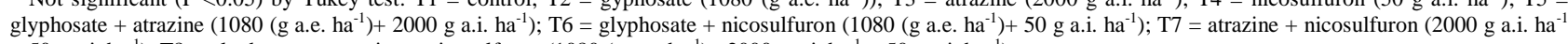
+50 g a.i. ha $\left.{ }^{-1}\right) ; \mathrm{T} 8=$ glyphosate + atrazine + nicosulfuron $\left(1080\left(\mathrm{~g}\right.\right.$ a.e. $\left.\mathrm{ha}^{-1}\right)+2000 \mathrm{~g}$ a.i. $\mathrm{ha}^{-1}+50 \mathrm{~g}$ a.i. ha $\left.{ }^{-1}\right)$

It is noteworthy that in the second experiment, some extra assessments that could not be measured in the first were done, achieving more concrete answers about the effects of treatments.

In both time points of experiments, a visual analysis of phytotoxicity was made, but not including notes since no significant symptoms were observed for treatments applied to the coloring and development of plants. This fact is related to the lack of differences in the assessments of chlorophyll indexes, in which there was a difference only in the first evaluation of SPAD at 14 DAA, while for all the other evaluations no differences were verified.

Application of nicosulfuron deserved a closer attention due to the damage that this herbicide can cause to the hybrid corn and hence we do not recommend the combination of the three herbicides used in this study. Recently, there are enough reports demonstrating that corn hybrids are more sensitive to nicosulfuron (Pereira Filho et al., 2000; Cavalieri et al., 2008; Cavalieri et al., 2010). Field technicians had access to this information more easily and had tables showing which materials are more sensitive (Vargas et al., 2006) but today results are missing on the positioning of nicosulfuron in new transgenic corn hybrids tolerant to glyphosate.

We highlight again that atrazine showed no damage to RR2 corn, corroborating with information on conventional corn (Dan et al., 2011). The glyphosate also did not bring any damage to the crop, corroborating studies that demonstrated the absence of glyphosate damage on RR2 corn, such as the effects on production and components at a dose of $1296 \mathrm{~g}$ a.i. $\mathrm{ha}^{-1}$ (Vieira Jr. et al., 2015) and when evaluating production components, nitrogen content, protein and oil in the grains, and shikimate levels in the plant, at doses up to $1296 \mathrm{~g}$ a.i. ha

${ }^{1}$ (Reddy et al., 2010). Some studies reported that glyphosate can cause problems for development for RR2 corn. This usually occurs with doses greater than those we applied in our studies (Albrecht et al., 2014).

With this information, we hoped that the real impact of the application of these herbicides on the development of RR2 corn can be better understood, aiming to provide conditions conducive to the sustainable use of these technologies and herbicides by farmers.

\section{Materials and methods}

The experiment was performed twice in a greenhouse. First, it was conducted in Piracicaba - SP, January-March 2014, in the greenhouse of the Department of Vegetables Production USP/ESALQ. Then it was repeated in Palotina - PR, JanuaryMarch 2015, in the greenhouse of the Department of Agronomic Sciences - UFPR/Setor Palotina, with the same treatments, but with simple hybrid corn showing distinct characteristics.

The experimental design was completely randomized, with four replications and eight treatments, totally 32 experimental units each time it was conducted. The treatments consisted of isolated and associated application of glyphosate, atrazine, nicosulfuron and an application without the treatment (control), as specified in Table 1.

Both times, the experiments were terminated at 28 days after application - DAA- near the V10 stage (Ritchie et al., 2001). The 7-liter vessels were used with one plant per pot and then kept at a temperature near $25^{\circ} \mathrm{C}$, relative humidity close to $60 \%$ air and irrigation of $5.0 \mathrm{~mm} \mathrm{day}^{-1}$.

The first time the experiment was conducted, the simple corn hybrid 30A91HR was used, and in the second time the simple corn hybrid 30F53HR. The two used hybrids are resistant to Lepidoptera, tolerance to glyphosate (Roundup Ready 2) and ammonium-glufosinate (LL), but LL technology is not commercially exploited in these hybrids. These hybrids were chosen for being suitable for the locations and mainly for displaying representative acreages in Brazil.

The positioning of fertilization practices, culture installation and plant health management systems were made according to the recommendations of EMBRAPA (2012). Fertilization was carried out taking into account the extraction of the culture, based on a yield of $10,000 \mathrm{~kg}$ ha- 1 . It is noteworthy that all the vessels were kept free from weed interference throughout the study period by means of manual control; thus isolating the effect of the herbicide on the corn plants.

Table 2 shows the results of the soil analysis used to fill the pots. The first time experiment was conducted (2014), in soil classified as typical dystrophic CLAYEY RED-YELLOW (EMBRAPA, 2013). In Table 3 the results of the soil analysis used for the second time the experiment has shown (2015), in which the soil classified as typical Eutroferric RED LATOSOL, with a very clayey texture (EMBRAPA, 2013). The application of the treatments was performed at the V4 phenological stage of corn cultivation (Ritchie et al., 2001). For this operation we used a $\mathrm{CO}_{2}$ backpack sprayer propelled 
at a constant pressure of $2 \mathrm{bar}$, with a flow of $0.65 \mathrm{~L} \mathrm{~min}^{-1}$, equipped with a bar containing 4 fan type tips, the Teejet series type XR 110.02, which worked at a height of $50 \mathrm{~cm}$ from the target and at speed of $1 \mathrm{~m} \mathrm{~second}^{-1}$, reaching an applied range $50 \mathrm{~cm}$ wide, providing a spray volume of $200 \mathrm{~L}$ $\mathrm{ha}^{-1}$. Regarding reviews at $7,14,21$ and 28 DAA the phytotoxicity was evaluated visually, considering visible symptoms on plants (SBCPD, 1995). Also at the same dates, plant height and stem diameter were evaluated. It is noteworthy that the first time that the experiment was conducted, we assessed stem diameter only at 28 DAA. The height measurement was taken from the soil surface to the opening of the cartridge, and the measuring of the stem diameter was performed four centimeters above the ground. Also at 7, 14, 21 and 28 DAA the chlorophyll content was evaluated. The first time that the experiment was carried out (2014) the portable meter SPAD-502 from Minolta was used. This instrument measures the intensity of the green in the leaf and calculates the SPAD (Soil Plant Analysis Development) that is highly correlated with the leaf chlorophyll content (Markwell et al., 1995). The second time that the experiment was conducted (2015) chlorophyll indexes types A, B and total were measured using an electronic chlorophyll meter (ClorofiLOG). This equipment determined the Falker chlorophyll index (FCI), which is highly correlated with laboratory analysis of chlorophyll types A and B (Falker, 2009). Chlorophyll levels were always evaluated in the first fully developed leaf. At $28 \mathrm{DAA}$, when the plants were near the V10 stage, experiments were then finalized with the measurement of fresh mass of the plant shoot and dry mass of the root system and from the shoot. For drying, the samples were placed at $65^{\circ} \mathrm{C}$ of forced ventilation for $72 \mathrm{~h}$ until obtaining constant mass. To measure the masses an analytical scale accurate to three decimal places were used. The data was analyzed according to Pimentel-Gomes and Garcia (2002), in which they met the basic assumptions for the variance analysis. The treatment averages were compared with the Tukey test $(P<0.05)$.

\section{Conclusions}

The applications of glyphosate and atrazine, isolated or in combination, did not result in damage to RR2 corn. This demonstrates the high selectivity of these hybrids in relation to these herbicides. Only nicosulfuron, showed the potential to cause damage to the crop, but this damage can be varied according to the corn hybrid used, in which some currently used hybrids had a lower selectivity with respect to this herbicide and sometimes in practical situations in the field the farmer does not perceive this deleterious effect.

\section{References}

Smith J, Jones MJ, Houghton LD (1999) Future of health insurance. N Engl J Med. 965:325-329.

Albrecht AJP, Albrecht LP, Barroso AAM, Victoria Filho R (2014) O milho RR2 e o glyphosate: Uma revisão. Rev. Br. Herbicidas. 13:58-67.

Azevedo LAS (2015) Mistura em tanque de herbicidas. In: Misturas de tanque de produtos fitossanitarios: teoria e prática. IMOS Graf. Ed. 1:89-113.

Barroso AAM, Albrecht AJP, Adegas FS, Alves PLCA, Toledo RE (2015) Manejo integrado do capim-amargoso. In: Barroso AAM, Alves PLCA (Org.) Biologia e manejo da resistência do capim-amargoso no Brasil. FUNEP 1: 4565 .
Borém A, Galvão JCC, Pimentel MA (2015) Milho: do plantio a colheita. UFV 1:351.

Cajacob CA, Feng PC, Reiser SE, Padgette SR (2007) Genetically modified herbicide resistant crops. In: Kramer W, Schirmer W (Ed.). Modern crop protection compounds. Wiley-vch :283-316.

Cavalieri SD, Oliveira Junior RS, Constantin J, Biffe DF, Rios FA, Franchini LHM (2008) Tolerance of corn hybrids to nicosulfuron. P Daninha. 26(1): 203-214.

Cavalieri SD, De Oliveira Junior RS, Constantin J, Biffe DF, Alonso DG, De Arantes JGZ, Canossa RS (2010) Contrastes entre procedências de híbridos de milho em relação à suscetibilidade aos herbicidas nicosulfuron e isoxaflutole. Semina. 31(4):811-822.

CELERES (2015) Informativo biotecnologia. 1:1-7.

CIB (2016) Eventos aprovados - CTNBio.

CONAB (2015) Acompanhamento da safra brasileira: grãos. $3: 147$.

Dan HA, Barroso ALDL, Braz GBP, Dan LGDM, Ferreira Filho WC, Menezes CCE, Azambuja US (2011) Seletividade do nicosulfuron e da mistura com atrazine na cultura do milho. Agrarian. 3(10):243-252.

EMBRAPA (2012) Sistema de Produção: cultivo do milho. 8 ed.

EMBRAPA (2013) Sistema brasileiro de classificação de solos. 3 ed. 353.

FALKER (2009) Medidor eletrônico de teor de clorofila clorofiLOG.

Gazziero DLP (2015) Misturas de agrotóxicos em tanque nas propriedades agrícolas do Brasil. P. Daninha 33(1):83-92.

Gemelli A, Oliveira Junior RS, Constantin J, Braz GBP, Jumes TMC, Gheno EAA, Rio FA, Franchini LHM (2013) Estratégias para o controle de capim-amargoso (Digitaria insularis) resistente ao glyphosate na cultura milho safrinha. Rev Br Herbicidas. 12(2):162-170.

Heap I (2016) International survey of herbicide resistant weeds.

Markwell J, Osterman JC, Mitchell JL (1995) Calibration of the Minolta SPAD-502 leaf chlorophyll meter. Photosynthesis Res. 46(3):467-472.

Melo MSC, Rosa LE, Brunharo CADG, Nicolai M, Christoffoleti PJ (2012) Alternativas para o controle químico de capim-amargoso (Digitaria insularis) resistente ao glyphosate. Rev Br Herbicidas. 2(11):195-203.

Pereira Filho IA, Oliveira MF, Pires NM (2000) Tolerância de híbridos de milho ao herbicida nicosulfuron. P Daninha. 18(3):479-482.

Pimentel-Gomes F, Garcia CH (2002) Estatística aplicada a experimentos agronômicos e florestais: exposição com exemplos e orientações para uso de aplicativos. FEALQ. $1: 309$.

Reddy KN, Bellaloui N, Zablotowicz RM (2010) Glyphosate effect on shikimate, nitrate reductase activity, yield, and seed composition in corn. J Agric Food Chem. 58(6):36463650.

Riar DS, Norsworthy JK, Steckel LE, Stephenson DO, Eubank TW, Bond J, Scott RC (2013) Adoption of best management practices for herbicide-resistant weeds in Midsouthern United States cotton, rice, and soybean. Weed Tech. 27(4):788-797.

Ritchie SW, Hanway JJ, Benson, GO (2001) Como a planta de milho se desenvolve. Informativo POTAFOS 15(103): 20.

Shaner DL (2000) The impact of glyphosate-tolerant crops on the use of other herbicides and on resistance management. Pest Manag. Sc. 56(4):320-326. 
SBCPD (1995) Procedimentos para instalação, avaliação e analise de experimentos com herbicidas. 1:42.

Vargas L, Peixoto CM, Roman ES (2006) Manejo de plantas daninhas na cultura de milho: principais herbicidas indicados para cultura de milho no sistema plantio direto e no preparo convencional do solo. Embrapa Trigo.
Vieira Júnior NS, Jakelaitis A, Cardoso IS, Rezende PN, Moraes NC, Araújo VT, Tavres CJ (2015) Associação de herbicidas aplicados em pós-emergência na cultura do milho. Glob Sc Tech. 8(1):1-8. 